REVIEW ARTICLE

\title{
Ureteral Injury During Colorectal Surgery: Two Case Reports and a Literature Review
}

\author{
Shlomo Yellinek, Dimitri Krizzuk, Juan J. Nogueras and Steven D. Wexner \\ Department of Colorectal Surgery, Cleveland Clinic Florida, Weston, FL
}

\begin{abstract}
:
Iatrogenic ureteral injury (IUI) is a dreaded complication of abdominopelvic surgery. Although rare, it is associated with severe consequences. This complication most commonly occurs during gynecological procedures but may also occur during colorectal surgeries. We present two cases of IUI in patients in whom the ureteric stents were electively placed. The first case was a 71-year-old male with no significant medical history. The patient underwent an elective laparoscopic sigmoidectomy for complicated diverticulitis. During the procedure, a proximal IUI occurred, and was recognized and repaired. The second case occurred in a 68 -year-old male with a history of multiple complicated abdominal surgeries. The patient underwent a second redo low anterior resection for a long preanastomotic stricture. The IUI occurred in the right fibrosed presacral plane, approximately $3 \mathrm{~cm}$ proximal to the bladder. The ureter was reimplanted to the bladder during the same procedure. We will also present a literature review of IUI, including the risk factors, intraoperative prevention, and repair options.
\end{abstract}

Keywords:

Iatrogenic ureteral injury, colorectal surgery, ureteral injury repair

J Anus Rectum Colon 2018; 2(3): 71-76

\section{Introduction}

Iatrogenic ureteral injuries (IUI) are known complications of abdominopelvic surgeries, which are associated with severe morbidity. Although rare, this serious complication may have serious adverse sequelae. Morbidity is reduced when the IUI is intraoperatively identified; unfortunately this occurs in only $15-30 \%$ of cases ${ }^{1-5}$. IUI most commonly occurs during gynecological procedures $(0.075-1.7 \%)$, followed by colorectal procedures $(0.25-1.1 \%)$. IUI during colorectal surgery most frequently occurs during proctectomy (abdominoperineal resection and low anterior resection) and sigmoidectomy ${ }^{6,7}$. The injury can be due to laceration, devascularization, ligation, thermal injury, or resection ${ }^{2-4,8-10}$.

We present two cases of IUI during colorectal surgery, which were intraoperatively identified and repaired. We will also discuss the risk factors for IUI, the means to prevent these types of injuries, intraoperative identification of IUI, and repair options.

\section{Case Presentation 1}

A 71-year-old male with no significant medical history was scheduled for elective laparoscopic sigmoidectomy eight weeks after resolution of an episode of perforated diverticulitis. After inducing general anesthesia, cystoscopy with insertion of bilateral ureteric stents was performed by the Urology Service. After mobilization of the left colon, the left ureter was identified as crossing the left iliac artery and continuing cephalad, seemingly into the retroperitoneum; the inferior mesenteric artery was then identified and divided. It appeared that the left ureter had been significantly medially deviated close to the proximal end of the left ureteral stent and was therefore transected with a bipolar energy device. 
The level of injury was at the proximal left ureter, close to crossing the gonadal vessels. The distal end of the ureter was completely transected and sealed. Gerota's fascia was identified and incised medially, after which the plane posterior to the ureters, between the psoas and the kidney, was developed. The left kidney was mobilized by dividing the lateral attachments and the hilum was identified. The renal pelvis was identified below the renal vein and was followed to the proximal cut edge of the ureter. The proximal and distal ureter was further mobilized, while still leaving periureteral tissue for blood supply. Both ends of the ureter were debrided and spatulated. The ureteroureteral anastomosis was performed using 4.0 polyglycolic acid interrupted sutures over a 5-French feeding tube. Once the posterior anastomosis was performed, a $6 \times 26$ double $\mathrm{J}$ ureteral stent was advanced proximally into the kidney and then distally into the bladder. The anterior portion of the anastomosis was completed with 4.0 polyglycolic acid interrupted sutures. The anastomosis appeared watertight. Jackson Pratt (JP) drains were left in the retroperitoneum. The patient was discharged home on the sixth postoperative day. The JP drain was removed after the creatinine levels reached a normal level and the patient was scheduled for elective removal of the ureteral double $\mathrm{J}$ stent in 6 weeks' time.

\section{Case Presentation 2}

A 68-year-old male with a history of multiple complicated abdominal surgeries revealed a previous left nephrectomy and partial gastrectomy for gastrointestinal stromal tumor and a previous anterior resection for diverticular disease followed by an anastomotic leak. Despite a redo anastomosis and loop ileostomy, he had a $12 \mathrm{~cm}$ long preanastomotic stricture and was scheduled to undergo elective laparotomy and second redo low anterior resection.

After inducing general anesthesia, cystoscopy with insertion of a right ureteric stent was performed by the Urology Service. Following extensive adhesiolysis, mobilization of the rectum began posteriorly and attempts were made to enter the significantly fibrosed presacral plane. As the dissection proceeded to the right, a right ureteric injury was confirmed by direct visualization of the ureteric stent.

Inspection revealed transection of the distal ureter approximately $3-4 \mathrm{~cm}$ proximal to the bladder. The ureter was difficult to identify due to significant fibrosis in the retroperitoneum. The ureter was moderately dilated down to an area of fibrosis in the deep pelvis approximately $4-5 \mathrm{~cm}$ from the bladder; the posterior bladder was also fibrotic. Anterior injury to the bladder was also noted. The proximal end of the ureter was difficult to identify and required mobilization near the kidney to gain sufficient length. The fibrotic area of the ureter was then excised to leave normal proximal ureter. The ureter was then spatulated and brought to the area of the bladder near the anterior wall where the bladder injury was noted. The ureter was anchored to the bladder using 4.0 polyglycolic acid sutures and a $6 \times 24 \mathrm{~cm}$ stent was then advanced into the ureter over a wire and the distal coil of the stent was manipulated into the bladder. The ureter was then anastomosed to the bladder using two layers of 4.0 polyglycolic acid sutures and a JP drain was left in the retroperitoneum.

The patient was discharged home on the seventh postoperative day and the JP drain was removed after his creatinine levels were normal. He was scheduled for elective removal of the bladder catheter in two weeks' time and removal of the ureteral double $\mathrm{J}$ stent in weeks weeks' time.

\section{Review of the Literature}

\section{Risk Factors for Ureteric Injury}

A recent population-based study by Halabi et al. ${ }^{2)}$ found an incidence of $0.28 \%$ IUI in more than two million colorectal surgeries in the USA. Patient risk factors for IUI included cancer with nodal or metastatic involvement, malnutrition, and steroid use. Disease risk factors were rectal cancer, followed by diverticular and Crohn's disease. Open surgery revealed higher rates of IUI compared to laparoscopic surgery; however, as there was no patient stratification, open cases presumably represent more difficult procedures due to reoperations and adhesions ${ }^{2}$. In contrast, Palanniapa et al. ${ }^{4}$ from Mount Sinai Medical Center reviewed 5729 colectomies for IUI. Conversely, in their series, there was a statistically significant increase in IUI occurring after laparoscopic versus open procedures $(0.66 \%$ vs. $0.15 \%$, respectively; $p=$ $0.007)^{4}$. Andersen et al. ${ }^{10)}$ reviewed the Danish National Colorectal Cancer database (DCCG) with 18,474 patients following resection for colorectal cancer. The rate of ureteral injuries in the entire cohort was $0.44 \%$, with $37(0.59 \%)$ injuries in the laparoscopic $(n=6,291)$ and $45(0.37 \%)$ in the open group $(n=12,183)(p=0.03)$. They divided their results into colon resections and rectal resections. No differences in the incidence of ureteral injury were found relative to the surgical approach in colon cancer patients. In rectal cancer patients $(n=5,959)$, the laparoscopic approach was used in 1899 patients, of whom 19 (1.0\%) sustained ureteral injuries, whereas $17(0.42 \%)$ of 4060 patients who underwent an open resection had a ureteral injury. In multivariate analysis adjusted for age, gender, ASA score, body mass index, tumor stage, preoperative chemoradiation, calendar year, and specialty of the surgeon, the laparoscopic approach was associated with an increased risk of ureteral injury (OR $=2.67 ; 95 \% \mathrm{CI}=1.26-5.65)^{10}$.

Prevention of Iatrogenic Ureteral Injury in Colorectal Surgery 
Table 1. Preventive Measures and Common Pitfalls of Iatrogenic Ureteral Injury (IUI).

Identification and reidentification of the ureter during each step of the dissection.

- It is not enough to identify the ureter in one location.

If necessary, convert the case to hand assisted or open.

Dissection of the "white line" on the colon side in order to leave the ureter in the retroperitoneum.

Know the common locations of ureteral injury.

In cases of inflammation or severe fibrosis, identify the ureter in an unaffected area.

The ureter may be displaced from its usual course in these cases.

Intraoperative diagnosis and treatment of IUI will have a more accurate prognosis, therefore, the surgeon must have a high level of suspicion regarding this injury.

The use of prophylactic ureteric stents is safe and useful.

Remember that the proximal part of the stent may not reach the proximal ureter.

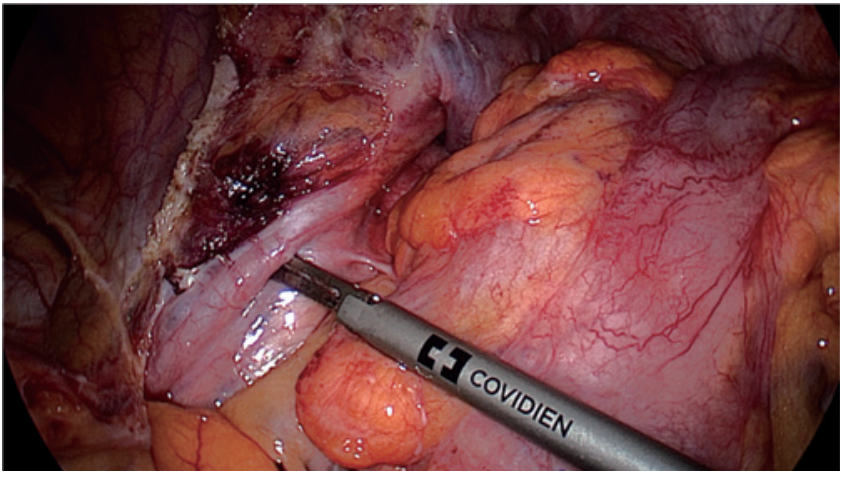

Figure 1. Left ureter in the left pelvic rim.

Table 1 summarizes the preventive measures as well as common pitfalls. Understanding of the anatomical course of the ureters and the adjacent organs is necessary in every lower abdominal surgery. Anatomically, the left ureter travels lateral to the sigmoid mesocolon and anterior to the psoas muscle and is crossed by the branches of the inferior mesenteric artery (IMA). The left ureter then takes a more medial course over the bifurcation of the left iliac vessels upon entering the pelvis. The right ureter travels lateral to the right mesocolon, anterior to the psoas muscle, and medially crosses above the bifurcation of the right iliac vessels. In females, the ureter crosses dorsal to the ovary, and underneath the broad ligament. In males, the vas deferens crosses ventral to the ureter. The ureters are divided into three anatomic segments: the upper segment extends from the ureteropelvic junction to the upper border of the sacroiliac joint; the middle segment courses over the sacrum; and the distal segment extends from the inferior border of the sacroiliac joint to the ureterovesical junction. Although may occur at any segment of the ureter, it most often occurs distally near the takeoff of the IMA or at the pelvic brim. Al-Awadi et al. ${ }^{5)}$ reported 82 cases of IUI over a period of 4 years. Anatomical locations were $65 \%, 20 \%$, and $15 \%$ for distal, mid, and proximal ureteral injury, respectively.

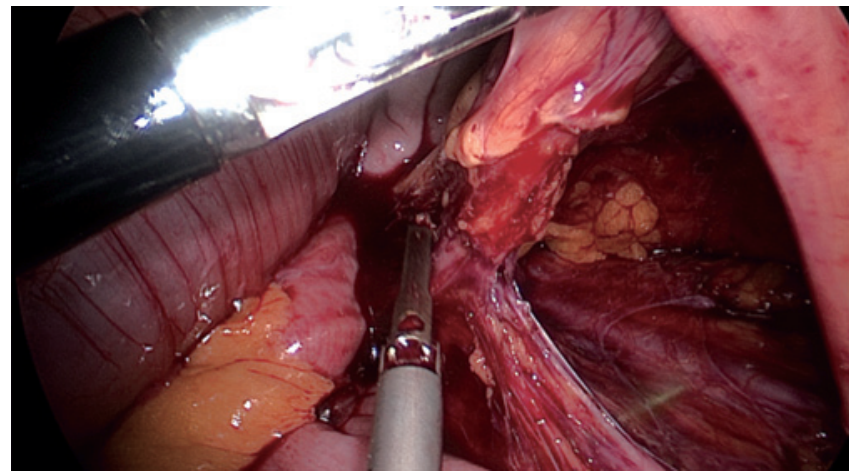

Figure 2. Left ureter seen before ligation of the inferior mesenteric artery.

The most important step in preventing IUI is exact identification of the ureter and careful dissection prior to resection of the mesocolon or colon. Identification should be under direct visualization and should be frequently repeated by either visualization of the "peristalsis" of the ureter by gently pressing on it or by feeling the "string." It is also possible to encircle the ureter with a loose tie or vessel loop to help reidentify it during the procedure. It is important to remember that the length of the ureter makes it susceptible to injury in multiple areas and during multiple steps of the surgery; thus, single identification is usually not sufficient. IUI injuries typically occur in three specific locations: at the takeoff of the IMA, at the pelvic brim, and between the lateral rectal ligaments ${ }^{11}$. Figure 1-3 demonstrates the common locations of ureteric injury.

When dissecting lateral-to-medial, the ureter should be identified at the pelvic brim and then dissection of the colon should be continued on the colon side of the white line. One pitfall in this step is dissecting the white line too laterally, which may cause mobilization of the ureter up to the mesocolon. During medial-to-lateral dissection, the ureter should again be identified through the parietal peritoneum prior to ligation of the IMA. The ureter can sustain injury during 


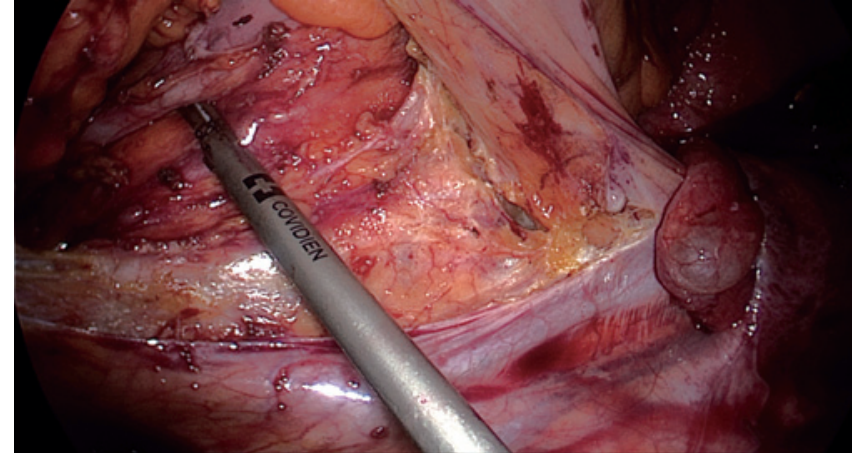

Figure 3. Bilateral ureters seen during dissection of the sigmoid mesocolon.

medial-to-lateral mobilization by mobilizing underneath it. If the ureter cannot be identified, additional efforts should be made such as converting the operation to an open or handassisted approach or redissecting from medial-to-lateral.

Anatomic variants, inflammation, prior irradiation, and reoperation may alter the usual course of the ureter; therefore, extra care must be taken to avoid IUI in these patients. Surgeons may consider obesity, inflammation, Crohn's disease, large tumors, reoperation, and prior pelvic irradiation risk as factors for IUI and, if so, may request ureteric stents. Prophylactic ureteric stents will not prevent IUI but will aid in intraoperative identification of the ureters, as was the case in both patients described in this report ${ }^{1,12}$. Our previous series published in 2007 retrospectively analyzed 313 patients undergoing laparoscopic colorectal procedures. Patients were divided into two groups: with and without ureteral stent insertion. Catheter insertion was more common in patients with diverticulitis, Crohn's disease, and chronic abscess; IUI was not found in either group. We concluded that prophylactic ureteric catheter placement in selected patients can be safely and successfully performed during laparoscopic colorectal surgery ${ }^{13)}$.

It is important to remember that the higher part of the stent may be in different anatomic locations, depending upon the anatomy of the patient and the urologist placing the stent. To date, there is no objective measure available to identify the exact height of the stent; therefore, ureteral injury proximal to the stent is a possibility. In the first patient described in this report, the proximal portion of the stent may have contributed to the ureteric angulation.

In cases where inflammation or fibrosis is noted during surgery, it is best to approach identification of the ureter from an unaffected area. Identification of the ureter may commence near the renal pelvis and continue distally. Moreover, converting to an open or hand-assisted procedure could be considered.

Recently, lighted ureteric stents (LUS) have been introduced to overcome the limitations of tactile feedback during minimally invasive surgery. Intuitively, this technique should prevent IUI by direct visualization of the ureters. Chahin et al. ${ }^{14)}$ evaluated 66 patients who underwent laparoscopic colorectal procedures and insertion of LUS. They reported one IUI that was conservatively managed by reinsertion of a left ureteral stent. The most frequent complication related to the stent insertion was gross hematuria in $65(98.4 \%)$ patients, which was conservatively managed without sequelae. The authors concluded that prophylactic LUS placement in laparoscopic colectomy is a safe procedure. Boyan et al. ${ }^{15)}$ reported a total of 465 laparoscopic colorectal resections with prophylactic LUS. No IUI occurred. Postoperative hematuria was present in $100 \%$ of patients and spontaneously resolved without sequelae. The authors confirmed that identification of the ureter via LUS can help prevent IUI in colorectal resections without significant morbidity. It was also advocated that LUS insertion might save time in the operating room by simplifying the ureter identification procedure and, in cases of injury, avoiding complex interventions. Considering the operative time required for injury identification and repair, the LUS insertion procedure itself is time-effective, requiring approximately 11-28 min, depending on the length of the procedure and coordination arrangements $\mathrm{s}^{6,14,15)}$.

There is concern that LUS generates excessive heat leading to urothelial injury and subsequent stricture formation. Pedro et al. ${ }^{16)}$ placed 3 different LUS in 9 female pigs, maintaining them in situ for $3 \mathrm{~h}$. During that time, luminescence was measured at $30 \mathrm{~min}$ intervals. At the end of the procedure, the authors measured the intraluminal temperature of the ureter and the tissue was harvested for histological evaluation. The authors concluded that there was no significant difference in temperature with the control group and the pathology report did not reveal any evidence of thermal injury to the urothelium or any remarkable alteration in the ureteral mucosa.

\section{Intraoperative Ureteral Injury Identification}

As briefly discussed, there is no high level evidence regarding the clinical value of prophylactic ureteric stents. Stents do not prevent ureteral injuries; however, they do aid in prompt intraoperative identification. Speicher et al. ${ }^{7}$ reviewed the NSQIP (ACS National Surgical Quality Improvement Program) database between 2005-2011 for all laparoscopic colectomies or proctectomies. Prophylactic ureteral stents were inserted in $4.2 \%$ of patients. Predictors for stent use were diverticular disease, radical resection, and recent radiotherapy. There was a slight increase in the operative time but no difference was observed in morbidity or mortality $^{7}$. Our group previously reported on 67 patients who had bilateral stent insertion, with no procedure-related morbidity and a lower rate of postoperative UTI $(1.5 \%)$ than in the control group $(2.5 \%)^{6}$.

If ureteric injury is suspected but cannot be proven, intravenous pyelogram, retrograde injection of methylene blue 
through the bladder, or intravenous injection may help. Indocyanine green (ICG) is a tricarbocyanine fluorophore that is gaining popularity for its variable uses in surgery. ICG may be used to highlight the ureter anatomy and can be a valid substitute to methylene blue, which is currently used, if IUI is suspected intraoperatively. In 2013, Lee et al. ${ }^{17}$ published an analysis of 7 patients undergoing robot-assisted ureteroureterostomy for ureteral stricture. ICG was used to identify the lesion and aid in the repair. It was concluded that intraureteral injection of ICG and visualization under near-infrared (NIR) fluorescent light aided in ureter delineation and identification of healthy versus diseased tissue ${ }^{17}$. The same technique can be transferred to ureter identification and IUI prevention. The method described by Siddighi et al. ${ }^{18)}$ involves a 6-French ureteral catheter cystoscopically inserted into the midureters and $10 \mathrm{~mL}$ of ICG injected retrograde into the lumen of each ureter. The ureteral catheters are clamped to minimize the leakage of ICG. Subsequent visualization under NIR fluorescence may be used as a means of primary and secondary prevention of IUI. With regards to secondary prevention, full-thickness ureteral injuries cause readily apparent leakage of ICG into the surgical field $^{18-20)}$. One possible drawback of intraureteral injection of ICG is its limitation for use in perfusion assessment due to subsequent low tissue contrast. It is also worth mentioning that the U.S. Food and Drug Administration (FDA) approval of ICG is limited for intravenous use; thus, it is imperative to disclose off-label intraureteral use ${ }^{19)}$. Additional fluorescent substances have been studied for that purpose. Dip et al. ${ }^{21)}$ was able to visualize the ureters in a rat model using sodium fluorescein after activation with a $530 \mathrm{~nm}$ light transmitter. Al-Taher et al. ${ }^{22}$ used intravenous methylene blue in 10 patients to identify the ureters, which were then visualized using a laparoscope with fluorescence imaging; however, the signal was detected after the ureter was already visible using the conventional light mode and the authors concluded that the technique did not provide any practical advantage over conventional laparoscopy.

One must remember that intraoperative identification and repair of IUI carries a more accurate prognosis than does delayed diagnosis and treatment. Al-Awadi et al. ${ }^{5)}$ found that the length of hospitalization, incidence of urinary leak, adhesions, and sepsis were significantly higher in the delayed diagnosis group.

\section{Repair of Ureteral Injuries}

Although usually performed by urologists, the colorectal surgeon should be familiar with the basic steps of IUI repair. The method of repair is influenced by the location of the injury and the extent of injured segment of the ureter. Minor contusions to the ureter can be treated with observation only or by ureteral stent insertion. Major lacerations, resection, or thermal injury will mandate anastomosis with de- bridement to the healthy proximal and distal tissue. The anastomosis is performed with absorbable sutures to prevent ureteral stone formation, creating a tension-free spatulated anastomosis over a ureteral stent, and a closed suction drain near the area of repair. In order to achieve this goal, mobilization of the kidney and/or the bladder may be needed. The stent will typically remain in situ for 4-6 weeks and will be removed after imaging studies confirm a patent anastomosis with no leak. The drain will be removed after confirmation of a leak-free anastomosis ${ }^{1,23,24}$.

Distal Ureteral Injuries

Repair options include ureteroureterostomy, ureterocystostomy, and psoas hitch. In general, if the injury is more than $3 \mathrm{~cm}$ from the ureterovesical junction, anastomosis repair can be performed between two parts of the ureter after mobilization of the ureter, adhering to the afore mentioned principles. If the injury is close to the bladder, reimplantation of the ureter into the bladder is the choice of repair, after mobilization of the proximal ureter. There are two repair options: one is intravesical repair, which entails tunneling of the reimplanted ureter into the bladder wall in order to prevent reflux. The other is extravesical repair that involves tunneling of the ureter through the detrusor muscle in order to prevent reflux. If ureterocystostomy cannot be achieved without tension, a psoas hitch should be considered, which involves mobilization of the bladder upward toward the proximal ureter. The bladder is mobilized by ligating the superior vesical pedicle on the contralateral side to the injury. The bladder is then opened through a transverse anterior cystotomy and secured to the psoas tendon. The ureter can then be tunneled in either an intra- or extravesical fashion, as described earlier. A ureteral stent is placed and the anterior cystotomy is closed in a vertical fashion ${ }^{11,23,24}$.

\section{Midureteral Injuries}

If the injured segment is short, an ureteroureterostomy can be performed; however, if the gap is long, either a psoas hitch or Boari flap may be considered. The latter involves raising the anterior bladder wall in a rectangular fashion and anchoring to the psoas tendon, as described earlier. The vascular supply of this flap is based on the ipsilateral superior vesical artery ${ }^{11,23,24}$.

\section{Proximal Ureteral Injuries}

A tension-free ureteroureterostomy is the preferred repair option and, moreover, may be aided by mobilization of the kidney to gain more length. In cases of a long gap, an ileal or appendiceal interposition can be used. This procedure is performed by anastomosing both ends of the ureter ends to the bowel. Crohn's disease and radiation enteritis are contraindications to this approach. In patients with very extensive severe injuries, a percutaneous nephrostomy or nephrectomy may be considered ${ }^{11,23,24}$. 


\section{Conclusion}

Although rare, IUI is a dreaded complication with high morbidity and associated costs. The long course of the ureter and its close proximity to other vital structures make this injury a risk factor in different anatomical locations as well as during different phases of the operation. The surgeon must be familiar with the anatomy of the ureter as well as the risk factors for injury. Furthermore, the surgeon must understand the therapeutic options following ureteric injury.

\section{Conflicts of Interest}

There are no conflicts of interest.

\section{References}

1. da Silva G, Boutros M, Wexner SD, Role of prophylactic ureteric stents in colorectal surgery. Asian J Endosc Surg. 2012 Aug; 5(3): 105-10.

2. Halabi WJ, Jafari MD, Nguyen VQ, et al. Ureteral injuries in colorectal surgery: an analysis of trends, outcomes, and risk factors over a 10-year period in the United States. Dis Colon Rectum. 2014 Feb; 57(2): 179-86.

3. Rao D, Yu H, Zhu H, et al. The diagnosis and treatment of iatrogenic ureteral and bladder injury caused by traditional gynaecology and obstetrics operation. Arch Gynecol Obstet. 2012 Mar; 285 (3): 763-5.

4. Palaniappa NC, Telem DA, Ranasinghe NE, et al. Incidence of iatrogenic ureteral injury after laparoscopic colectomy. Arch Surg. 2012 Mar; 147(3): 267-71.

5. Al-Awadi K, Kehinde EO, Al-Hunayan A, et al. Iatrogenic ureteric injuries: incidence, aetiological factors and the effect of early management on subsequent outcome. Int Urol Nephrol. 2005 Jun; 37 (2): 235-41.

6. Nam YS, Wexner SD. Clinical value of prophylactic ureteral stent indwelling during laparoscopic colorectal surgery. J Korean Med Sci. 2002 Oct; 17(5): 633-5.

7. Speicher PJ, Goldsmith ZG, Nussbaum DP, et al. Ureteral stenting in laparoscopic colorectal surgery. J Surg Res. 2014 Jul; 190(1): 98-103.

8. Léonard F, Fotso A, Borghese B, et al. Ureteral complications from laparoscopic hysterectomy indicated for benign uterine pathologies: a 13-year experience in a continuous series of 1300 patients. Hum Reprod. 2007 Jul; 22(7): 2006-2011.

9. Ozdemir E, Ozturk U, Celen S, et al. Urinary complications of gynecologic surgery: iatrogenic urinary tract system injuries in obstetrics and gynecology operations. Clin Exp Obstet Gynecol. 2011 Jan; 38(3): 217-20.
10. Andersen P, Andersen LM, Iversen LH. Iatrogenic ureteral injury in colorectal cancer surgery: a nationwide study comparing laparoscopic and open approaches. Surg Endosc. 2015 Jun; 29(6): 140612 .

11. Delacroix SE, Winters JC. Urinary tract injures: recognition and management. Clin Colon Rectal Surg. 2010 Jun; 23(2): 104-12.

12. Bothwell WN, Bleicher RJ, Dent TL. Prophylactic ureteral catheterization in colon surgery. A five-year review. Dis Colon Rectum. 1994 Apr; 37(4): 330-4.

13. Tsujinaka S, Wexner SD, DaSilva G, et al. Prophylactic ureteric catheters in laparoscopic colorectal surgery. Tech Coloproctol. 2008 Mar; 12(1): 45-50.

14. Chahin F, Dwivedi AJ, Paramesh A, et al. The implications of lighted ureteral stenting in laparoscopic colectomy. JSLS 2002 Jan-Mar; 6(1): 49-52.

15. Boyan WP, Lavy D, Dinallo A, et al. Lighted ureteral stents in laparoscopic colorectal surgery; a five-year experience. Ann Transl Med. 2017 Feb; 5(3): 44.

16. Pedro RN, Kishore TA, Hinck BD, et al. Comparative analysis of lighted ureteral stents: lumination and tissue effects. J Endourol. 2008 Nov; 22(11): 2555-8.

17. Lee Z, Simhan J, Parker DC, et al. Novel use of indocyanine green for intraoperative, real-time localization of ureteral stenosis during robot-assisted ureteroureterostomy. Urology. 2013 Sep; 82 (3): 729-33.

18. Siddighi S, Yune JJ, Hardesty J. Indocyanine green for intraoperative localization of ureter. Am J Obstet Gynecol. 2014 Oct; 211 (4): 436.e1-2.

19. Lee Z, Kaplan J, Giusto L, et al. Prevention of iatrogenic ureteral injuries during robotic gynecologic surgery: a review. Am J Obstet Gynecol. 2016 May; 214(5): 566-71.

20. Tanaka E, Ohnishi S, Laurence RG, et al. Real-time intraoperative ureteral guidance using invisible near-infrared fluorescence. J Urol. 2007 Nov; 178(5): 2197-202.

21. Dip FD, Nahmod M, Anzorena FS, et al. Novel technique for identification of ureters using sodium fluorescein. Surg Endosc. 2014 Sep; 28(9): 2730-3.

22. Al-Taher M, van den Bos J, Schols RM, et al. Fluorescence ureteral visualization in human laparoscopic colorectal surgery using methylene blue. J Laparoendosc Adv Surg Tech A. 2016 Nov; 26(11): 870-5

23. Lynch TH, Martínez-Piñeiro L, Plas E, et al. EAU guidelines on urological trauma. Eur Urol. 2005 Jan; 47(1): 1-15.

24. Morey AF, Brandes S, Dugi DD $3^{\text {rd }}$, et al. Urotrauma: AUA guideline. J Urol. 2014 Aug; 192(2): 327-35.

Journal of the Anus, Rectum and Colon is an Open Access article distributed under the Creative Commons Attribution-NonCommercial-NoDerivatives 4.0 International License. To view the details of this license, please visit (https://creativec ommons.org/licenses/by-nc-nd/4.0/). 